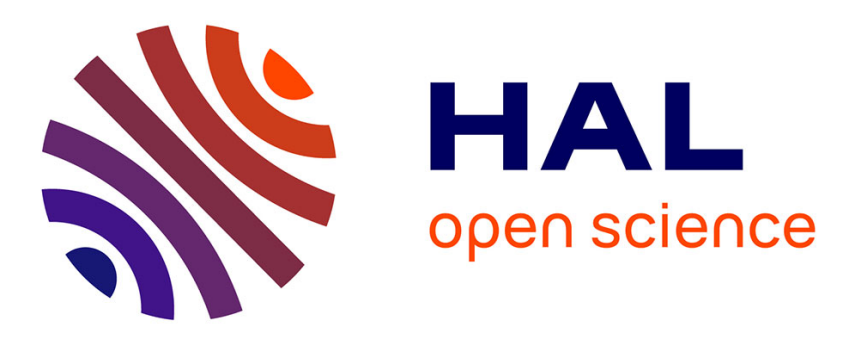

\title{
Growth activity during fingering in a porous Hele Shaw cell
}

\author{
Grunde Løvoll, Yves Méheust, Renaud Toussaint, Jean Schmittbuhl, Knut
}

Jørgen Måløy

\section{- To cite this version:}

Grunde Løvoll, Yves Méheust, Renaud Toussaint, Jean Schmittbuhl, Knut Jørgen Måløy. Growth activity during fingering in a porous Hele Shaw cell. Physical Review E: Statistical, Nonlinear, and Soft Matter Physics, 2004, 70 (2), pp.026301. 10.1103/physreve.70.026301 . hal-00110571

\section{HAL Id: hal-00110571 \\ https://hal.science/hal-00110571}

Submitted on 20 Nov 2018

HAL is a multi-disciplinary open access archive for the deposit and dissemination of scientific research documents, whether they are published or not. The documents may come from teaching and research institutions in France or abroad, or from public or private research centers.
L'archive ouverte pluridisciplinaire HAL, est destinée au dépôt et à la diffusion de documents scientifiques de niveau recherche, publiés ou non, émanant des établissements d'enseignement et de recherche français ou étrangers, des laboratoires publics ou privés. 


\title{
Growth activity during fingering in a porous Hele-Shaw cell
}

\author{
Grunde Løvoll, ${ }^{1,2}$ Yves Méheust, ${ }^{3,2,1}$ Renaud Toussaint, ${ }^{1,3}$ Jean Schmittbuhl, ${ }^{2}$ and Knut Jørgen Måløy ${ }^{1}$ \\ ${ }^{1}$ Department of Physics, University of Oslo, Norway \\ ${ }^{2}$ Laboratoire de Géologie, École Normale Supérieure, Paris, France \\ ${ }^{3}$ Department of Physics, NTNU Trondheim, Norway
}

(Received 9 October 2003; revised manuscript received 10 February 2004; published 9 August 2004)

\begin{abstract}
We present in this paper an experimental study of the invasion activity during unstable drainage in a two-dimensional random porous medium, when the (wetting) displaced fluid has a high viscosity with respect to that of the (nonwetting) displacing fluid, and for a range of almost two decades in capillary numbers corresponding to the transition between capillary and viscous fingering. We show that the invasion process takes place in an active zone within a characteristic screening length $\lambda$ from the tip of the most advanced finger. The invasion probability density is found to only depend on the distance $z$ to the latter tip and to be independent of the value for the capillary number $\mathrm{Ca}$. The mass density along the flow direction is related analytically to the invasion probability density, and the scaling with respect to the capillary number is consistent with a power law. Other quantities characteristic of the displacement process, such as the speed of the most advanced finger tip or the characteristic finger width, are also consistent with power laws of the capillary number. The link between the growth probability and the pressure field is studied analytically and an expression for the pressure in the defending fluid along the cluster is derived. The measured pressure is then compared with the corresponding simulated pressure field using this expression for the boundary condition on the cluster.
\end{abstract}

DOI: 10.1103/PhysRevE.70.026301

PACS number(s): 47.20.Gv, 47.55.Mh, 47.54.+r, 47.55.-t

\section{INTRODUCTION}

Different types of unstable fluid displacements in porous media play an important role in many natural and commercial processes [1,2]. Development of a better understanding of these processes therefore has a broad scientific interest as well as potentially huge economical benefits. The complex patterns observed in such processes have been extensively studied and modeled over the last decades; see [1-7] and references therein.

The geometry of the displacement structures observed in immiscible two-phase flow is in general controlled by the competition between viscous forces, gravitational forces, and capillary forces; those various forces act on scales ranging from the pore scale to the system size. The relative wettabilities, viscosities, and densities of the fluids, as well as the heterogeneity of the underlying porous media, play an important role in the competition process. The relative magnitudes of viscous and capillary forces (on pore scale) are quantified through the dimensionless capillary number $\mathrm{Ca}$ $=\left(\mu_{w} v_{f} a^{2}\right) /(\gamma \kappa)$ where $\mu_{w}$ is the viscosity in the wetting (displaced) fluid, $v_{f}$ is the filtration speed, $a$ is the characteristic pore size, $\gamma$ is the interface tension, and $\kappa$ is the permeability of the porous medium.

In this paper we address a drainage experiment in which nonwetting air displaces a high-viscous wetting glycerin/ water solution in a horizontal two-dimensional porous medium; hence, gravity has no influence on the displacement. The porous medium consists of a Hele-Shaw cell filled with a random monolayer of monodisperse glass beads. We investigate the crossover regime between the regime of slow displacement for which capillary forces control the dynamics of the invasion process and the geometry of the resulting invasion structure (capillary fingering), and that of fast displace- ments where viscous forces are dominant (viscous fingering). We emphasize on the dependence of the invasion probability density $\phi$, or activity, on the distance to the most advanced finger tip along the interface. The invasion probability density $\phi$ is the growth probability of the invasion structure; it is fundamental because both the structure and dynamics are controlled by this function. Growth probability has been discussed extensively in the past for diffusion limited aggregation (DLA) simulations [8-13] where it was found to be the multifractal distribution of the harmonic measure [11-14]. A strong analogy exists between the structures obtained by DLA and viscous fingering, as was first pointed by Paterson [15]. Both processes obey the Laplacian growth equation

$$
\begin{gathered}
\nabla^{2} P=0, \\
v \propto-\nabla P,
\end{gathered}
$$

where $P$ denotes the diffusing field-i.e., the probability density of random walkers in DLA or the pressure in viscous fingering - and $v$ denotes the speed of the interface. However, both processes differ in that accreting particles of fixed size are added one by one in DLA, at a random location set by the growth probability proportional to $-\nabla P$, whereas in an empty Hele-Shaw cell, which is a regular porous medium of constant permeability, the growth process is deterministic and full layers are invaded along the whole interface, with a local velovity set by $-\nabla P$. In the absence of surface tension, Mullins-Sekerka instabilities develop in the deterministic Laplacian growth problem, leading to cusp singularities of the interface in a finite time [16]. This instability is regularized by the smallest scales accessible to the system, and another difference between DLA and viscous fingering in regular porous media is the nature of this so-called ultraviolet regularization, set by surface tension in viscous fingering or by the 
particle size in DLA. The boundary value at the interface is given by surface tension $\gamma$ for viscous fingering, $P \propto-\gamma / r$, where $r$ is the local curvature of the boundary between both fluids, in contrast to DLA where it is set to constant $P$. These differences lead to very different structures in channel geometry-namely, stationary solutions corresponding to a Saffman-Taylor regular interface propagating at constant velocity in the case of viscous fingering in Hele-Shaw cells $[7,17,18]$, as opposed to branched structures in the case of DLA $[19,20]$. In radial geometries however, solutions of both problems display branching and tip splitting, and some authors have argued that none of the above-mentioned differences affect the large scale structure and that viscous fingering patterns are identical to coarse grained DLA clusters [21]. Using the recently developed Hastings-Levitov formalism of iterated conformal maps [22], the relationship between DLA and deterministic Laplacian growth has been intensely investigated [22-28]. Although the issue for Laplacian growth is still controversial [24-26], it seems that DLA, Laplacian growth and viscous fingering in Hele-Shaw cells display indeed the same large-scale structure in radial geometry, with fractal dimensions respectively determined as $D=1.713 \pm 0.0003$ [29], $D=1.7$ [27], and $D=1.70 \pm 0.03$ [17].

The process of viscous fingering during drainage in a random porous medium, under study in the present paper, is $a$ priori distinct from the above cases: although the pressure field satisfies the Laplace equation (1) at large scales, the presence of glass beads in the Hele-Shaw cell affects the viscous fingering process, modifying significantly the pressure boundary condition along the interface between the two fluids, with respect to the empty Hele-Shaw cell case. Fundamentally, the local interface curvature controlling the capillary pressure drop depends on the local pore geometry and is independent of the large-scale curvature, and distributed sizes of pore throats lead to a random distribution of capillary pressure thresholds inside the porous medium. These capillary pressure threshold values introduce a lower cutoff for the invasion probabilities, even for fast flows. In the slow displacement limit for which $v_{f} \simeq 0$, the invasion process is entirely controlled by the fluctuations of the capillary threshold distribution inside the porous medium [6,30]. For finite displacement velocities, as this study will show, the growth process is in this case intermediate between the one-by-one feature of DLA and the layer-by-layer characteristic of Laplacian growth: in the system studied here, several pores along the interface are invaded simultaneously, although not all of them-see Fig. 3-and it takes a finite time to invade a full pore. This pore-scale randomness in viscous fingering results in branched structure as well in channel geometry, as shown on Fig. 2, in contrast with the Saffman-Taylor fingers obtained in empty Hele-Shaw cells [7]. This might also be the reason why the measured fractal dimensions of viscous fingering patterns in radial geometry are reported slightly lower in random porous media similar to the one used here, $D=1.62$ [31], than in empty Hele-Shaw cells, $D=1.70$ [18].

Imbibition experiments (wetting fluid displacing a nonwetting fluid) were previously performed in a quasi-twodimensional system [32,33], where the width of the viscous fingers was measured to scale with the capillary number as $[32,33] w_{f} \propto \mathrm{Ca}^{-0.5}$. This scaling relation was explained by a

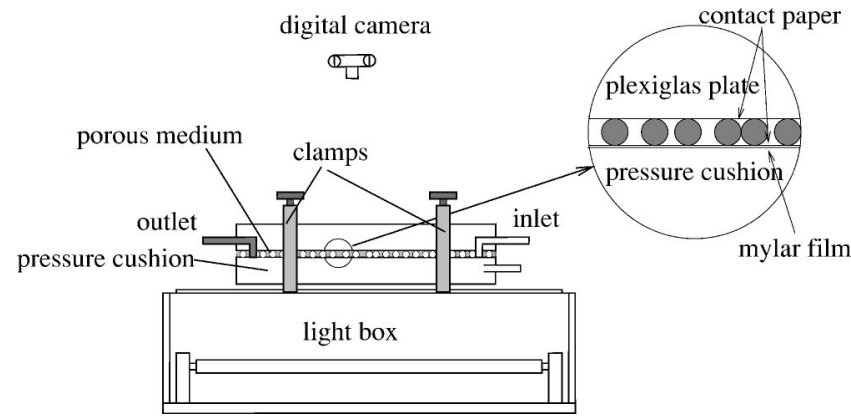

FIG. 1. Sketch of the experimental setup with the light box for illumination, the porous model, and the digital camera. The porous medium is sandwiched between two contact papers and kept together with a "pressure cushion."

strong dynamic component of the capillary pressure [33]. We do not observe a strong dynamic component of the capillary pressure in our experiments (see below). The geometry of the invader for drainage is also significantly different from the invader structure of imbibition [32-35].

In this study we study experimentally the growth probability density $\phi(z)$ as a function of the distance $z$ (in the flow direction) from the most advanced finger tip and its dependence on the extraction speed (or capillary number). We also investigate experimentally the mass density $n(z)$ along the flow direction of the invader and confront the behavior of the measured $\phi(z)$ and $n(z)$ to what we expect from analytical arguments. A calculation of the $z$ dependence of the pressure on the surface of the invader is presented, which yields the $\mathrm{z}$ dependence of the capillary pressure and shows a direct link with the measured growth probability density. Pressure measurements are performed in the model and compared with pressures simulated by solving the Laplace equation with this pressure boundary condition. Other features characteristic of the displacement, such as outermost tip velocity and the width of the invasion fingers, are also investigated.

The present article is organized as follows. We first present the experimental method (Sec. II). We then discuss the experimental results (Sec. III), and prospects (Sec. IV) before concluding (Sec. V).

\section{EXPERIMENTAL METHOD}

The experimental setup is shown in Fig. 1. The porous model consists of a monolayer of glass beads of diameter $a$ $=1 \mathrm{~mm}$ which is randomly spread between two contact papers $[31,36]$. The model is a transparent rectangular box of dimensions $L \times W$ and thickness $a$.

Two models of widths $W=430 \mathrm{~mm}$ and $W=215 \mathrm{~mm}$ have been used in the experiments; their other characteristics were identical. They are respectively referred to in the rest of the article as the "wide" and "narrow" models.

To prevent bending of the model a 2-cm-thick glass plate and a 2-cm-thick Plexiglas plate are placed on top of the model. To squeeze the beads and the contact paper together with the upper plate, a Mylar membrane mounted on a 2.5$\mathrm{cm}$-thick Plexiglas plate, below the model, is kept under a 
3.5-m water pressure as a "pressure cushion." The upper and lower plates are kept together by clamps, and the side boundaries are sealed by a rectangular silicon rubber packing. Milled inlet and outlet channels are made in the upper Plexiglas plate. The distance between the inlet and outlet channels defines the length of the model, $L=840 \mathrm{~mm}$. One should also note that a few beads are removed from a small region near the center of the inlet channel, to initiate the invader in the center of the inlet. This is done to avoid edge effects appearing when the invader grows to the lateral boundaries of the model. The porosity of the models is measured to be 0.63 and the permeability is $\kappa=(0.0166 \pm 0.0017) \times 10^{-3} \mathrm{~cm}^{2}$ $=(1685 \pm 175)$ darcy.

The defending wetting fluid used in all our experiments is a $90 \%-10 \%$ by weight glycerol-water solution dyed with $0.1 \%$ Negrosine to increase the contrast between the colored fluid and the invader. Air is used as the invading nonwetting fluid. The wetting glycerol-water solution has a viscosity of $\mu_{w} \approx 0.165 \mathrm{~Pa} \mathrm{~s}$ and a density of $\rho_{w}=1235 \mathrm{~kg} \mathrm{~m}^{-3}$ at room temperature. The corresponding parameters for the nonwetting air are $\mu_{n w}=1.9 \times 10^{-5} \mathrm{~Pa} \mathrm{~s}$ and $\rho_{n w}=116 \mathrm{~kg} \mathrm{~m}^{-3}$. The viscous ratio is thus $M=\mu_{n w} / \mu_{w} \sim 10^{-4}$. The surface tension between these two liquids is $\gamma=6.4 \times 10^{-2} \mathrm{~N} \mathrm{~m}^{-1}$. The temperature in the defending fluid is controlled and measured at the outlet of the model during each experiment, so as to accurately estimate the viscosity of the wetting fluid.

The absolute pressure in the wetting liquid is measured in the outlet channel and at a point at a distance of $280 \mathrm{~mm}$ (in the flow direction) from the inlet channel and $38 \mathrm{~mm}$ from the left boundary (looking in the flow direction) using Honeywell 26PCA Flow-Through pressure sensors.

The invader is visualized by illuminating the model from below with a light box and pictures are taken at regular intervals with a Kodak DCS 420 CCD camera, which is controlled by a computer over a SCSI connection. This computer records both the pictures and the pressure measurements. Each image contains $1536 \times 1024$ pixels, which corresponds to a spatial resolution of $0.55 \mathrm{~mm}$ per pixel or $\sim 3.22$ pixels per pore $\left(1 \mathrm{~mm}^{2}\right)$; the color scale contains 256 gray levels. The gray level distribution of the image presents two peaks corresponding, respectively, to the white air-filled and dark gray glycerol-filled parts of the image. The image is filtered so as to obtain a clear boundary between the two phases, through a scheme that mainly consists in removing the background and thresholding at a gray level value between the two latter peaks. All further image treatments are performed on the resulting black and white image.

To check possible dynamic components of the capillary pressure we performed gravity stabilized experiments by keeping the experimental model vertical [36] and extracting the glycerol/water mixture from the bottom of the model. The capillary pressure was measured by recording the pressure in the model as the stabilized fluid front approaches the sensor. No systematic dynamic effect on the capillary pressure was found. For the low injection rates the width of the fronts was further used to estimate the minimum and width $W_{c}$ of the capillary pressure threshold distribution.

Throughout the paper the following coordinate system is used: $\left(x, z^{\prime}\right)$ is the orthonormal frame describing the porous medium plane, with $z^{\prime}$ the spatial coordinate in the direction
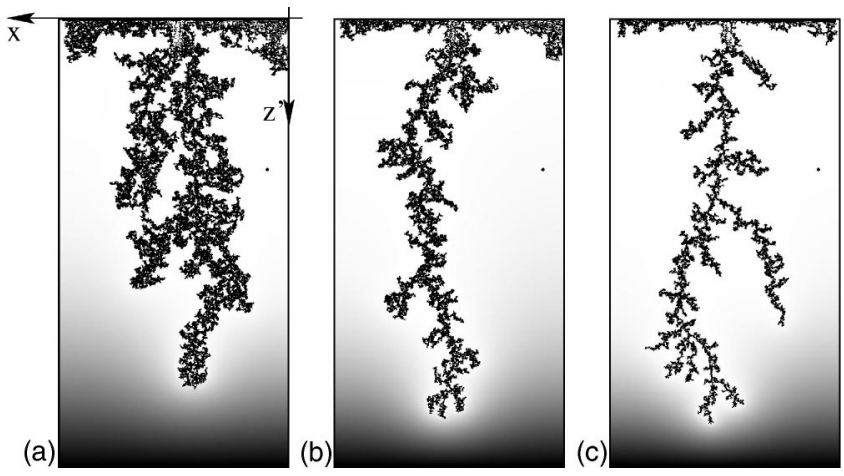

FIG. 2. Displacement structures obtained for different withdrawal rates: (a) $\mathrm{Ca}=0.027$, (b) $\mathrm{Ca}=0.059$, and (c) $\mathrm{Ca}=0.22$. The images have been treated to separate the two phases. The black frame denotes the outer boundaries of the model, while the black spot close to the right edge of the model denotes the position of the pressure sensor. The simulated pressure field is shown superimposed on the image. Dark shadings correspond to low pressures while light shadings correspond to high pressure.

of the flow (positive in flow direction). The position of the most advanced finger tip is denoted $z_{\text {tip }}^{\prime}$; its speed along the $z^{\prime}$ axis is denoted $v_{t i p}=\dot{z}_{t i p}^{\prime}$. The position along the $z^{\prime}$ axis computed with respect to that of the most advanced finger tip is $z=z_{\text {tip }}^{\prime}-z^{\prime}$. Those coordinates are indicated in Figs. 2 and 3.

\section{RESULTS}

We present 12 experiments using the wide model for values of the capillary number $\mathrm{Ca}$ ranging from $1.4 \times 10^{-2}$ to $3.6 \times 10^{-1}$ and 5 experiments using the narrow model for capillary numbers ranging from $3.3 \times 10^{-2}$ to $1.9 \times 10^{-1}$. The latter series was conducted to check system size dependences. For every experiment, we have carefully investigated the invasion process.

Figure 2 displays air clusters observed for the same porous medium, at three different flow rates. The complex structure of the air clusters is drawn in black. The particles of the porous medium are not shown. The defender-i.e., the glycerin/water solution - is drawn using a scale of grays ranging from white around the air cluster to black near the setup outlet; these shadings represent the intensity of the numerically estimated pressure field in the defending fluid (see Sec. III A). Figure 2(a) addresses an experiment carried out at a small rate $(\mathrm{Ca}=0.027)$. It displays a fat cluster where capillary forces dominate the dynamics of the invasion process, leading to a so-called capillary fingering. At larger displacement rates $[\mathrm{Ca}=0.22$, Fig. 2(c)], the "fingers" appear thinner and less internal trapping of the defender is observed. This regime is dominated by viscous forces and is generally labeled as viscous fingering. For intermediate capillary numbers $[\mathrm{Ca}=0.059$, Fig. 2(b)], the aspect of the cluster includes both geometries: capillary fingering at small scale and viscous fingering at large scale.

The pressure field around the clusters exhibits a fingerlike structure that has analogies to Saffman-Taylor fingers, as will 


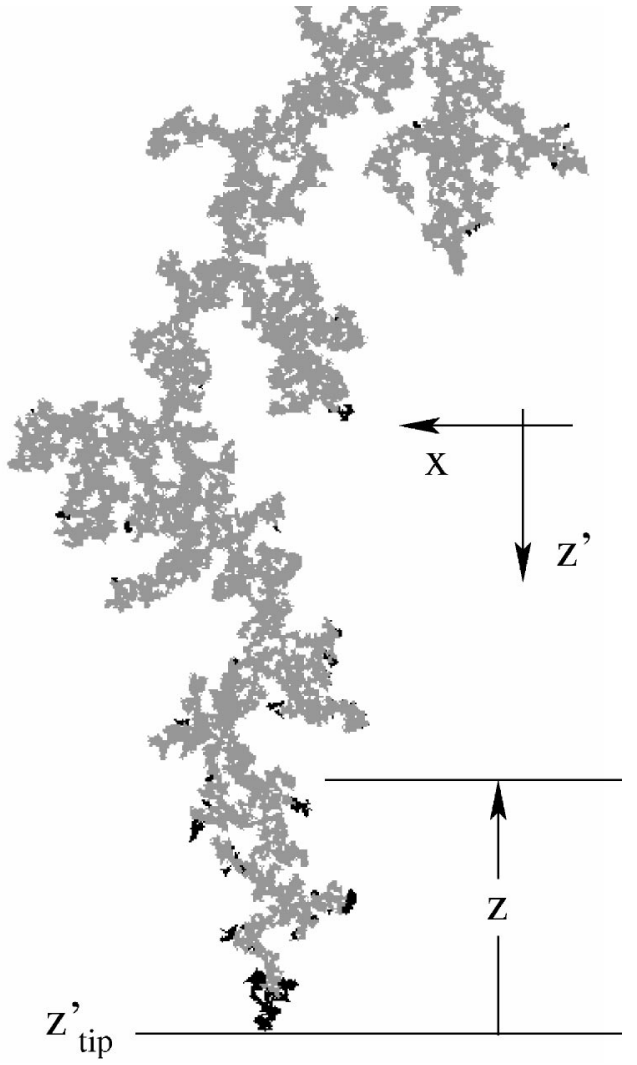

FIG. 3. Two consecutive images taken at a time interval $\Delta t$ $\simeq 15$ during the experiment at $\mathrm{Ca}=0.059$, drawn on top of each other. The invaded regions in the first image are painted light gray, the growth areas obtained by subtracting the first picture from the other one are painted black. The coordinate system used throughout the paper is also shown.

be discussed in Sec. IV. Accordingly, the displacement exhibits obvious capillary-number-dependent features, which are discussed in detail in Sec. III B below. In Sec. III A, we focus on the relation between the growth activity, the frozen structure left behind, and the pressure field in front of the fingers.

\section{A. Relation between growth activity, frozen structure, and fluid pressures}

The growth activity has been investigated by measuring the growth probability density $\phi(z)$ from series of images and performing pressure measurements.

\section{Definition of growth probability density $\phi(z)$ and mass density $n(z)$}

To investigate the growth process, images have been taken with constant time delay $\Delta t$ between each image. The tip position of the longest finger is identified to find the coordinate system $(x, z)$ and to be able to calculate the speed of the longest finger. The differential growth between two images is found by a direct image subtraction between two subsequent images. After the subtraction we typically have a collection of invaded pores representing the growth (see Fig.

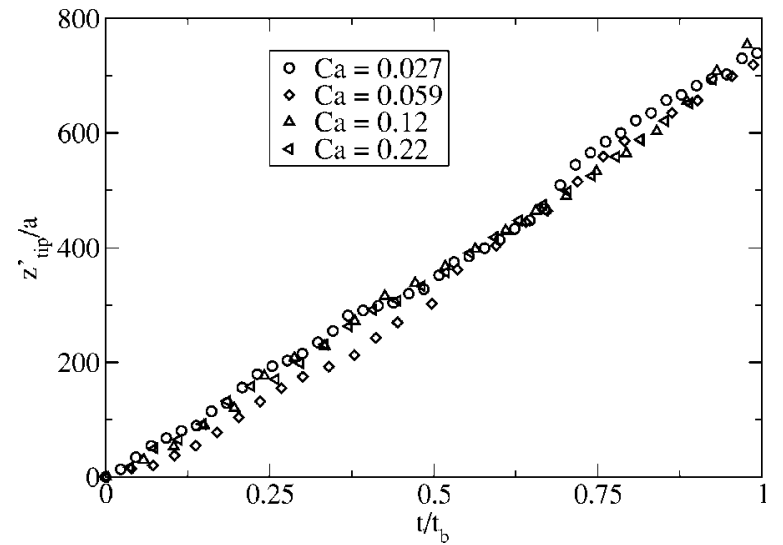

FIG. 4. Plot of the position of the most advanced finger tip, $z_{\text {tip }}^{\prime}$, as a function of time. Data from the wide model. The time is rescaled by dividing with the break through time $t_{b}$, which is the time the most advanced finger reaches the outlet channel. The values of $t_{b}$ are $5347 \mathrm{~s}, 1204 \mathrm{~s}, 476 \mathrm{~s}$, and $256 \mathrm{~s}$ for capillary numbers $\mathrm{Ca}$ equal to $0.027,0.059,0.12$, and 0.22 , respectively.

3). The growth density $\phi^{*}(z)$ is defined as the average number of filled pores within $[z, z+\Delta z]$ divided with $\Delta z$. After an initial regime corresponding to the time needed for the longest finger to propagate a distance of the order of the width of the porous medium $W, \phi^{*}(z)$ is found to be fairly independent of time up to a few percents variations. In a given experiment with constant $\mathrm{Ca}, \phi^{*}(z)$ is then averaged over all images excluding this initial regime, to obtain a good average of the stationary growth function. The growth probability density $\phi(z)=K \phi^{*}(z)$, where $K$ is a normalization constant, is then found by normalizing $\phi^{*}(z)$ with respect to $z$ so that

$$
\int_{0}^{L} \phi(z) d z=1
$$

Note that, in the remainder of the paper, $z$ is in units of pore size $(a=1 \mathrm{~mm})$.

The mass density of the frozen structure $n(z)$ is defined as the average number of filled pores within $[z, z+\Delta z]$ divided with $\Delta z$. The average is taken over all images in a given experiment with constant $\mathrm{Ca}$. Both $n(z)$ and $\phi(z)$ appeared to be fairly robust with respect to the width $\Delta z$ of the analysis strips used to compute them.

\section{Growth activity and the frozen structure left behind}

For all experiments, the speed of the most advanced finger tip was observed to be fairly constant. Figure 4 shows the position of the most advanced finger tip, $z_{\text {tip }}^{\prime}$, for different capillary numbers. After a short initial stage, the speed of the fingers saturates to a constant average value. Linear fits to the behavior $z_{\text {tip }}^{\prime}$ as a function of time outside the initiation stage provide an average finger tip speed $v_{t i p}$ for all experiments.

The measured invasion probability density function $\phi(z)$ is plotted in Fig. 5 as a function of the distance to the finger tip for the two system sizes on a linear-logarithmic plot. An exponential-like decay is seen for $z<W / 2$ with a deviation 

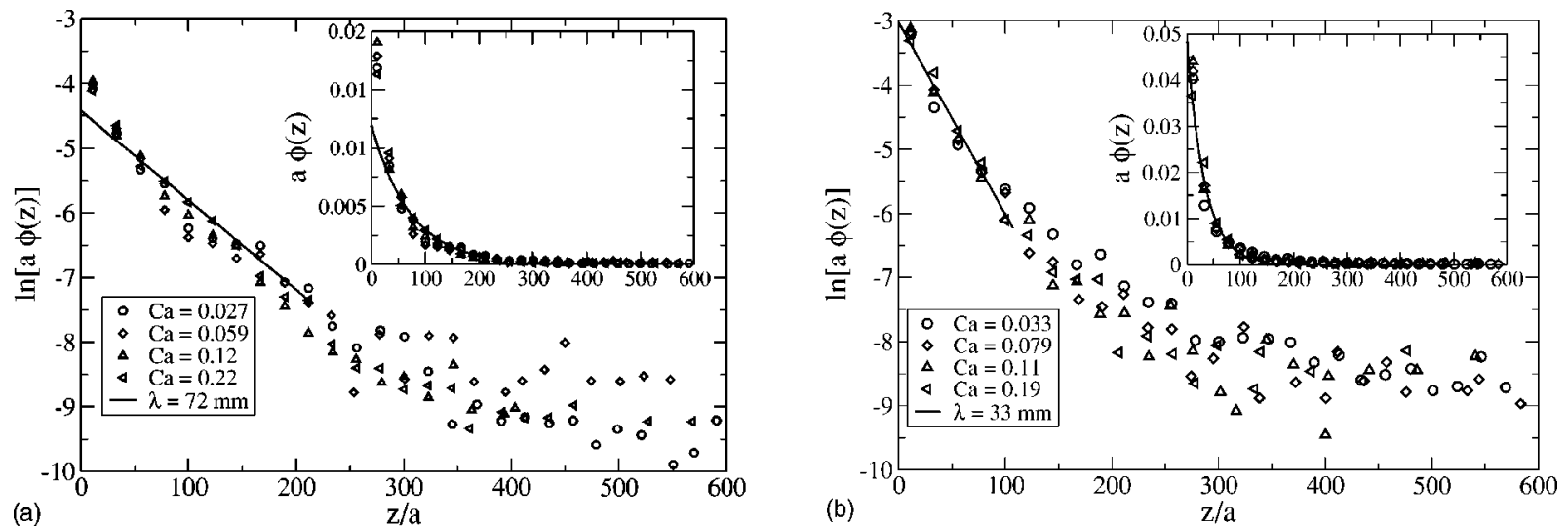

FIG. 5. Linear-logarithmic plot of the invasion probability density $\phi(z)$ as a function of the distance $z$ to the finger tip (insets are double linear plots of the same invasion probability density). (a) Data from the wide experimental model and (b) from the narrow model. The solid lines in the curves correspond to the model function $\ln \left(B e^{-z / \lambda}\right)$ obtained from linear regression over $\ln [a \cdot \phi(z)]$ for $z<W / 2$, where $\phi(z)$ is the average measured invasion probability density. Note that $\phi(z)$ are averages of all the datasets and not only the sets shown in the graphs above. The average screening length $\lambda$ is estimated from this fit.

from exponential behavior for larger lengths. A characteristic decay length or "screening length" $\lambda$ is estimated from linear fits to the linear-logarithmic data for $z<W / 2$ (see Fig. 5). As we can see from these plots a nice data collapse is obtained, indicating that the invasion probability density $\phi(z)$ and, thus, the screening length $\lambda$ are independent of the capillary number for a given system. On the other hand, when comparing the two systems, the screening length $\lambda$ depends on the system size: $\lambda=(72 \pm 10) \mathrm{mm}$ for the wide model and $\lambda$ $=(33 \pm 5) \mathrm{mm}$ for the narrow model. The actual shape of function $\phi$ also seems to be weakly dependent on the system size.

Relating the mass of the frozen structure, $n(z)$, to the invasion probability density $\phi(z)$ and confronting the obtained relation to experimental results provides new insights into the displacement process. The total number of invaded pores in a time interval $[t, t+\Delta t]$ is $R \Delta t$, where $R$ is the number of invaded pores per time unit. For a given flow rate $Q, R$ is related to $Q$ and to the characteristic pore volume $V_{\text {pore }}$ by the relation $Q=R V_{\text {pore }}$, so that

$$
R=\frac{W}{a^{2}} v_{f},
$$

where $W$ is the width of the system, $a$ is the characteristic pore size, and $v_{f}$ is the Darcy or filtering velocity of the wetting fluid; for a given porous medium and fluid pair $v_{f}$ $\propto \mathrm{Ca}$. The number of invaded pores in the analysis strip defined by $z \in[z, z+\Delta z]$ during time interval $[t, t+\Delta t]$ is then $R \Delta t \phi(z) \Delta z$. The tip position $z_{\text {tip }}^{\prime}$ is further given by $z_{\text {tip }}^{\prime}(t)$ $=z_{0}^{\prime}+v_{\text {tip }} t$ where $v_{\text {tip }}$ is the speed of the finger tip (assumed to be constant) and $z_{0}^{\prime}=z_{\text {tip }}^{\prime}(t=0)$. This is a fairly good approximation after a short initial regime as seen in Fig. 4.

The total number of invaded pores in an analysis strip of width $\Delta z$ at a distance $z$ from the finger tip is thus given by

$$
n(z) \Delta z=R \int_{t_{0}}^{t} \phi\left[z\left(t^{\prime}\right)\right] \Delta z d t^{\prime},
$$

where $t_{0}$ is the time at which $z_{\text {tip }}^{\prime}=z^{\prime}$. Taking advantage of the linear relation between the coordinate $z$ and tip speed $v_{\text {tip }}$, $z(t)=z_{\text {tip }}^{\prime}(t)-z_{\text {tip }}^{\prime}\left(t_{0}\right)=\left(t-t_{0}\right) v_{\text {tip }}$, Eq. (5) becomes

$$
n(z)=\frac{R}{v_{\text {tip }}} \int_{0}^{z} \phi(\widetilde{z}) d \widetilde{z} \equiv \frac{R}{v_{\text {tip }}} \Phi(z) .
$$

Using Eq. (4) we finally obtain the relation between the linear density of invaded pores (or "cluster-mass" density), $n(z)$, to the cumulative invasion probability density distribution $\Phi(z)$ :

$$
n(z)=n_{\mathrm{Ca}} \Phi(z),
$$

where

$$
n_{\mathrm{Ca}}=\frac{W \gamma \kappa}{\mu_{w} a^{4}} \frac{\mathrm{Ca}}{v_{\text {tip }}} .
$$

The characteristic average mass density can also be related to the filtration velocity: $n_{\mathrm{Ca}}=\left(W / a^{2}\right)\left(v_{f} / v_{\text {tip }}\right)$. Equation (7) is confirmed by Fig. 6, where $n(z) / n_{\mathrm{Ca}}$ is plotted as a function of $z / a$. All experimental plots collapse, confirming that there is one single cumulative probability distribution $\Phi(z)$ for the system for all experiments at different extraction speeds. The function $\Phi(z)$, computed as an average function from all cumulative probability functions for the various experiments, is plotted in Fig. 6 as a plain line. Note that $n_{\mathrm{Ca}}$ and consequently $n(z)$ are capillary number dependent, which is directly visible on Fig. 2. The explicit dependence of the saturation mass density on $\mathrm{Ca}$ will be plotted in Fig. 9(a). The inset of Fig. 6 shows $1-n(z) / n_{\mathrm{Ca}}$ on a linear-logarithmic scale. The solid lines represents $1-\Phi(z)$ and the dashed lines $1-e^{-z / \lambda}$ which would be the model function for a pure exponential $\phi$ ( $\lambda$ is the screening length evaluated before).

From the results presented above we conclude that the active invasion zone is defined by a screening length $\lambda$ which is constant for a given porous media and liquid pair and at a range of capillary numbers of two decades. However, we expect this result to be valid only for sufficiently high filtration speed $v_{f}$. Indeed, on the one hand, the capillary fingering regime $(\mathrm{Ca} \simeq 0)$ corresponds to an invasion that is controlled by fluctuations in the capillary threshold pressures, so that 

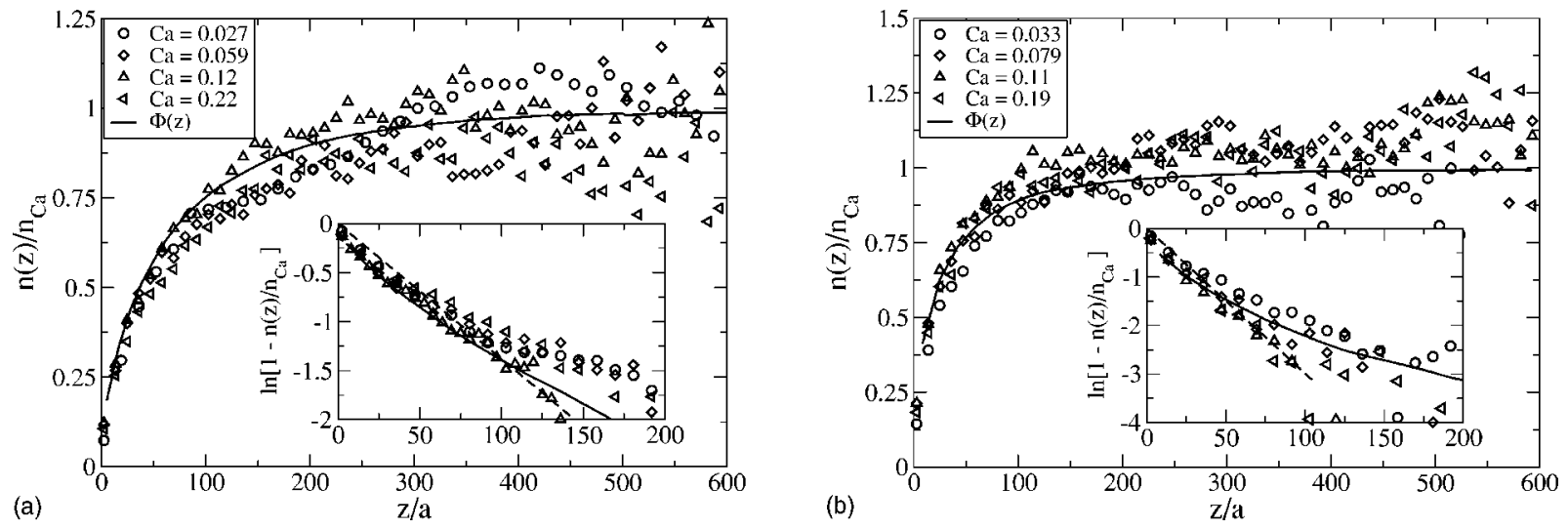

FIG. 6. Plot of the rescaled average mass density of nonwetting fluid $n(z) / n_{\mathrm{Ca}}$, where $n_{\mathrm{Ca}} \propto \mathrm{Ca} / v_{\mathrm{tip}}$, inside the model as a function of the distance to the tip of the most advanced finger $z$ : (a) the wide model and (b) the narrow model. The average cumulative invasion probability function $\Phi(z)$ is plotted on top of the curves for comparison in the two cases. The insets of (a) and (b) show the plots $\ln \left[1-n(z) / n_{\mathrm{Ca}}\right]$ as a function of $z$. The solid lines in the insets correspond to $\ln [1-\Phi(z)]$, and the dashed lines have the slope $1 / \lambda$ where $\lambda$ is the screening length of $\phi(z)$ found from Fig. 5-i.e., respectively, $\lambda=(72 \pm 10) \mathrm{mm}$ and $(33 \pm 5) \mathrm{mm}$ for the wide model and narrow model.

invasion occurs along the whole front [6,30]. There is no well-defined finger tip or growth direction in that limit. The width of the capillary threshold pressure distribution $W_{c}$ is larger than the viscous pressure drop over the whole system and defining a screening length or active zone is not meaningful.

When the length of the system is larger than its width, it is found from both pressure measurements and simulations that the decay in pressure into the structure from the longest finger occurs on a length scale of the order of the width of the system (see Fig. 2). We therefore expect $W$ and not $L$ to be the relevant length scale for the decay of the pressure field close to the tip. Viscous forces can therefore be considered to dominate capillary pressures if the following criteria are met:

$$
W_{c}<\frac{W \mu v_{f}}{\kappa},
$$

or if we assume $W_{c} \sim\left\langle P_{c}\right\rangle \approx \gamma / a$, where $\left\langle P_{c}\right\rangle$ is the average capillary pressure (which is the case here):

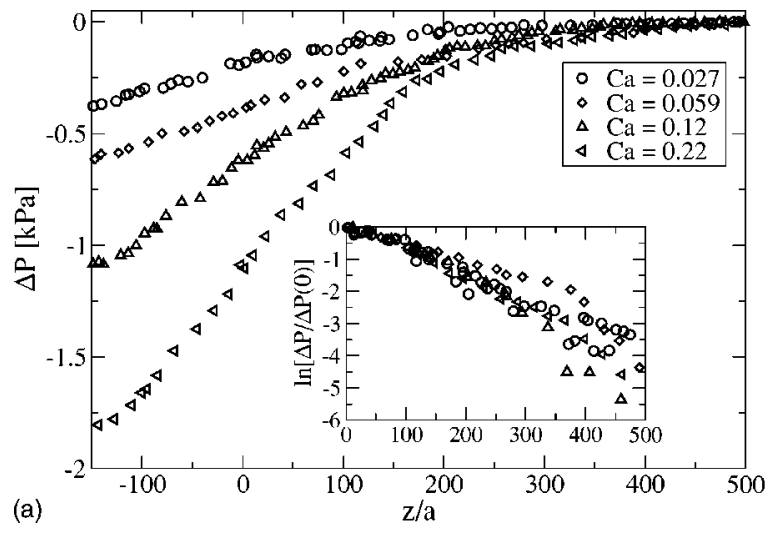

$$
\mathrm{Ca}>a / W .
$$

For our system $a / W \sim 10^{-3}$, which is an order of magnitude smaller than our lowest capillary number.

On the other hand, for situations where the "pure viscous fingering" in a random porous media has been reached, there is no trapping of wetting liquid inside the fingers, which reached the lower one-pore width limit (at $\mathrm{Ca} \approx 0.2$ in our system). Whether the screening length or active zone has the same width or behaves identically as for lower capillary numbers is not clear. We believe that the screening by the most advanced finger is a viscous effect, which remains important as the displacement speed increases. In this one-pore limit, however, the tip speed dependence on the capillary number is modified, as will be further detailed in Sec. III B.

\section{Relation between the growth probability density $\phi(z)$ and the fluid pressure}

Figure 7 shows the dependence of the pressure difference $\Delta P(z)=P(z)-P(\infty)$ in the wetting liquid as a function of the distance $z$ to the outermost tip for different capillary num-

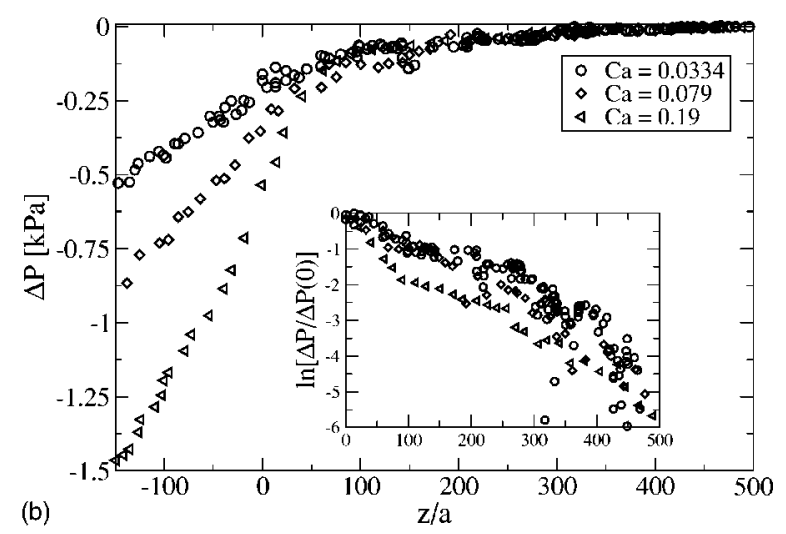

FIG. 7. Plot of the pressure difference $\Delta P(z)=P(z)-P(\infty)$ measured by the pressure sensor located in the position $\left(x_{s}, z_{s}^{\prime}\right)$ $=(38 \mathrm{~mm}, 280 \mathrm{~mm})$. The inset is the same data plotted in a semilogarithmic plot where $P(z)$ is scaled with $\Delta P(0)=P(z=0)-P(\infty)$ to illustrate the decay of the pressure field. (a) is for the wide model and (b) is for the narrow model. 
bers. Here $P(\infty) \equiv P_{0}-P_{c}(\infty)$ is the liquid pressure along the interface far behind the finger tip, with $P_{0}$ the pressure in the nonwetting liquid and $P_{c}(\infty)$ the capillary pressure in this stagnant zone. It is important to note that the pressure $P(z)$ is measured on the side of the model (indicated in Fig. 2) while the fingers are propagating in the central part of the model (Fig. 2).

The pressure seems to be linearly dependent on the distance from the tip during a first stage before the tip reaches the sensor. In a second stage, after the finger tip has passed the sensor, pressure relaxes and reaches the value $P(\infty)$. A closer inspection of the pressure curves (see Fig. 7) shows that there is no clear systematic dependence of the pressure relaxation on the capillary number. The pressure difference $P(z)-P(\infty)$ decays with approximately the same length for the different capillary numbers (see inset of Fig. 7). This indicates that the details of the internal structure of the "fingers" do not have a strong influence on the pressure field on large scales.

The pressure measurements are related to the invasion activity by the following considerations. Let us consider the local speed of an interface located in an arbitrary pore throat between two pores, one filled with air and the other with the wetting liquid. Let $P(x, z)$ be the pore pressure in the wetting liquid and $P_{t}(x, z)$ be the capillary pressure threshold value to invade that pore. Note that this is different from the pressure $P(z)$ defined as the pressure measured on the side of the model at the sensor position. The pore throat at position $(x, z)$ is passed under the condition that the pressure difference $P_{c}(x, z)=P_{0}-P(x, z)$ is larger than the capillary threshold pressure $P_{t}(x, z)$ at this position. If invasion occurs, a characteristic value of the speed of the interface will be

$$
v(x, z)=\frac{2 \kappa}{\mu} \frac{P_{0}-P(x, z)-P_{t}(x, z)}{a} .
$$

In this equation we have used the permeability of the porous media $\kappa$ as an approximation for the average single pore permeability to get the right order of magnitude. Let $N\left(P_{t}(x, z)\right)$ be the capillary pressure distribution. For the sake of simplicity, we assume a flat capillary pressure distribution with lower limit $P_{t}^{\min }$, upper limit $P_{t}^{\max }$, and width $W_{c}$. Under the condition that the viscous pressure drop over a pore $a$ does not exceed $W_{c}$ (which we have checked by means of numerical simulations), the expectational value of the interface velocity (average value over the capillary threshold distribution), while the pore is getting invaded, will be

$$
\begin{aligned}
\langle v(x, z)\rangle= & \frac{1}{P_{0}-P(x, z)-P_{t}^{\min }} \int_{P_{t}^{\min }}^{P_{0}-P(x, z)} \frac{2 \kappa}{a \mu}\left[P_{0}-P(x, z)\right. \\
& \left.-P_{t}(x, z)\right] d P_{t} \\
= & \frac{\kappa}{a \mu}\left[P_{0}-P(x, z)-P_{t}^{\min }\right] .
\end{aligned}
$$

Here, $P_{t}^{\min }$ is the minimum of the distribution for capillary threshold; when $P_{0}-P(x, z)$ goes to that minimum, the expectational value for the speed of the interface goes to zero. The growth probability density $\phi(x, z)$ for the invasion struc- ture within a time $[t, t+\Delta t]$ at a position $(x, z)$ is proportional to $\langle v(x, z)\rangle$ times the probability $p(x, z)$ that the throat gets invaded; hence,

$$
\phi(x, z)=C\langle v(x, z)\rangle p(x, z),
$$

where $C$ is a normalization constant, which we can find by integrating the above equation along the invasion front:

$$
\begin{gathered}
\int_{S} \phi(x, z) d l=C \int_{S}\langle v(x, z)\rangle p(x, z) d l \\
1=\frac{C}{a} \int_{S} a\langle v(x, z)\rangle p(x, z) d l=\frac{C}{a} Q,
\end{gathered}
$$

where $Q$ is the flow rate, and thus

$$
C=\frac{a}{Q} .
$$

Since we have assumed a flat capillary threshold distribution of width $W_{c}$, the probability that the pore at position $(x, z)$ gets invaded is

$$
p(x, y)=\frac{1}{W_{c}}\left[P_{0}-P(x, z)-P_{t}^{\mathrm{min}}\right] .
$$

From Eqs. (12)-(17) we obtain, for the growth probability density $\phi(x, z)$ in position $(x, z)$,

$$
\phi(x, z)=\frac{\kappa}{Q \mu W_{c}}\left[P_{0}-P_{t}^{\min }-P(x, z)\right]^{2} .
$$

Averaging this expression over $x$ and introducing the number of interface sites at a distance $z$ from the tip, $f(z)$, we obtain the invasion probability density $\phi(z)$ as

$$
\begin{gathered}
\phi(z)=f(z)\langle\phi(x, z)\rangle_{x}, \\
\phi(z)=f(z) \frac{\kappa}{Q \mu W_{c}}\left[P_{0}-P_{t}^{\min }-\langle P(x, z)\rangle_{x}\right]^{2},
\end{gathered}
$$

for which we have assumed that $P(x, z)$ is a function of $z$ only (lowest-order approximation). Equation (20) yields

$$
\langle P(x, z)\rangle_{x}=P_{0}-P_{t}^{\min }-\left(\frac{\phi(z)}{f(z)} \frac{Q W_{c} \mu}{\kappa}\right)^{1 / 2},
$$

which can be rewritten by introducing the relation between the flow rate and the capillary number. Accordingly the average pressure in the wetting fluid in the immediate vicinity of the interface and at position $z$ is related to the activity $\phi(z)$ according to

$$
\langle P(x, z)\rangle_{x}=P_{0}-P_{t}^{\min }-\left(\operatorname{Ca} \gamma W_{c} \frac{W}{a} \frac{\phi(z)}{f(z)}\right)^{1 / 2} .
$$

Let us now look closer at the "snapshots" of the experiments shown in Fig. 2. For $z=0$, the last correction term in Eq. (22) is $170 \mathrm{~Pa}$ for the fastest experiment $(\mathrm{Ca}=0.22)$ and $65 \mathrm{~Pa}$ for the slowest experiments $(\mathrm{Ca}=0.027)$. At the same moment, the imposed external pressures in the outlet channel are $3055 \mathrm{~Pa}$ for the fastest and $625 \mathrm{~Pa}$ for the slowest experi- 


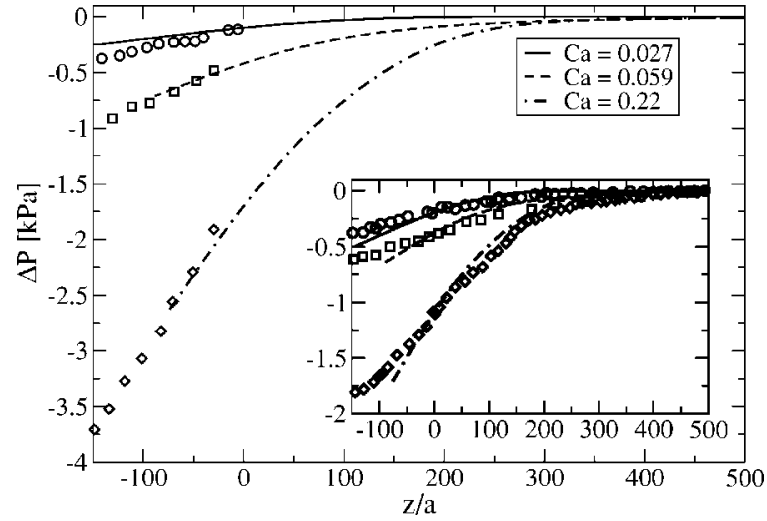

FIG. 8. The simulated pressure along the line with $x_{s}=38 \mathrm{~mm}$ (corresponding to the $x$ coordinate of the pressure sensor) for the invasion structures in Fig. 2. The data points in the main graph show the corresponding pressures measured at the outlet sensor. In the inset we plot scaled simulation data with corresponding pressure data measured inside the model at $\left(x_{s}, z_{s}^{\prime}\right)=(38 \mathrm{~mm}, 280 \mathrm{~mm})$.

ments. The minimum capillary pressure is estimated to $373 \mathrm{~Pa}$ and the width of the capillary distribution to $200 \mathrm{~Pa}$. This indicates that the correction term in Eq. (22) should not be neglected. In Fig. 2 is shown the gray-scale map of the pressure field at a particular time, simulated from the displacement structures obtained experimentally. A very strong screening is seen for all injection rates. The large-scale structure of the pressure field in the vicinity of tip of the longest finger looks visually very similar even if the invader structure is quite different. In the simulations of the pressure field we have used Eq. (22) to set the proper boundary conditions. The pressure field has been calculated by solving the Laplace equation for the pressure using a conjugate gradient method [37]. We used the boundary condition given by Eq. (22) on the cluster and the inlet line. As boundary condition on the outlet we used the pressure $P(\infty)-\Delta P_{\text {tot }}$, where $-\Delta P_{\text {tot }}$ is the total viscous pressure drop imposed in the corresponding experiment at that moment. To obtain $P(\infty)=P_{0}-P_{c}(\infty)$, the capillary pressure $P_{c}(\infty)$ was measured in the experiments for large values of $z$. Figure 8 shows the simulated pressure $\Delta P\left(x_{s}, z\right)=P\left(x_{s}, z\right)-P(\infty)$ as a function of the $z$ coordinate relative to the tip position defined as previously, at a fixed lateral position $x_{s}$ corresponding to the $x$ coordinate of the pressure sensor. It is important to note that this is somewhat different from the experiments since the pressure is measured at different $z^{\prime}$ positions, but at the same time-i.e., with a fixed geometry of the invasion cluster-while in the experiments the pressure is measured at a fixed $z^{\prime}$ position, at different times corresponding to various stages of the invasion cluster. The length scale of the decay of the pressure for $z>0$ is very similar in the experiments and the simulations (see comment below). However, the pressure difference $\Delta P(z)$ in the simulations is lower than the $\Delta P(z)$ measured by the sensor [at position $\left.\left(x_{s}, z_{s}^{\prime}\right)=(38 \mathrm{~mm}, 280 \mathrm{~mm})\right]$ in the model (see Fig. 7). This is due to the strong boundary effects of the pressure close to the outlet channel: the tips in the simulations situations are very close to this boundary along which the pressure is fixed (see Fig. 2), while in the situations corresponding to the measurements at $z<0$ plotted in Fig. 7, the outlet boundary was far ahead of the finger tip, and the pressure boundary condition was equivalent to an imposed gradient at infinite distance. To check the importance of this boundary effect on the magnitude of the pressure difference, the simulated pressure has been compared with the pressure difference $\Delta P(z)$ evaluated from measurements at the outlet channel, as the finger tip progressed further than the stage corresponding to the simulations. The agreement in Fig. 8 between the simulated pressure and the data points corresponding to the outlet channel measurements is then satisfactory.

To compare the length scale of the decay of the pressure for $z>0$ between the simulations and the experiment, we then compare the pressure data measured inside the model at the sensor position to the simulation data scaled by a factor such as $\Delta P\left(x_{s}, z=0\right)$, which would be equal in experiments and simulations. Such a rescaling of the simulation pressure profile simply corresponds to the result of an identical simulation still carried on the invasion clusters of Fig. 2, with identical boundary conditions derived from the growth density function for the pressure along the clusters, but where the imposed pressure along the bottom boundary is such that the pressure at point $\left(x_{s}, z_{\text {tip }}^{\prime}\right)$ would coincide with the pressure measured in the experiments when the tip passed at the same height as the sensor--i.e., when $z_{\text {tip }}^{\prime}=z_{s}^{\prime}$. This ensures that the pressure gradient and pressure value in the region around the tip of the invading cluster are of the same order in these rescaled simulations and in the experimental stages corresponding to $z \sim 0$ in Fig. 7, which is a first-order technique to correct for the strong boundary effect and compare with these experimental situations where the bottom boundary is much farther away. The pressure measured in the experiments at sensor position and this scaled simulation data are plotted in the inset of Fig. 8. This comparison shows that the decay in the pressure happens at comparable length scales in the simulations and experiments.

Eventually, the local structure of the finger and the lateral $x$ distance from the invader to the pressure sensor will also have an important influence on the pressure field. The difference in the pressure field between the left and the right side of the finger (looking in the flow direction) in Fig. 2 illustrates this point. The deviation between the experimental data points and the simulations for the lowest capillary number of the main part of Fig. 8 may be explained by this effect. As the lateral position $x_{\text {tip }}$ of the invading structure moves during the experiment and is importantly varying from an experiment to the next, this effect also explains the important dispersion of the scaled pressure drops $\Delta P(z) / \Delta P(0)$ observed in the inset of Fig. 7(b).

\section{B. Capillary-number-dependent features}

As stated in the introduction to Sec. III, Fig. 2 clearly shows that some features of the invading cluster depend on the capillary number. The mass $n(z)$ of the invasion cluster obviously decreases with increasing capillary number; in relation to this, the speed of the most advanced finger tip, $v_{\text {tip }}$, increases with the capillary number, and there is a systematic trend for fingers to become thinner as capillary number in- 

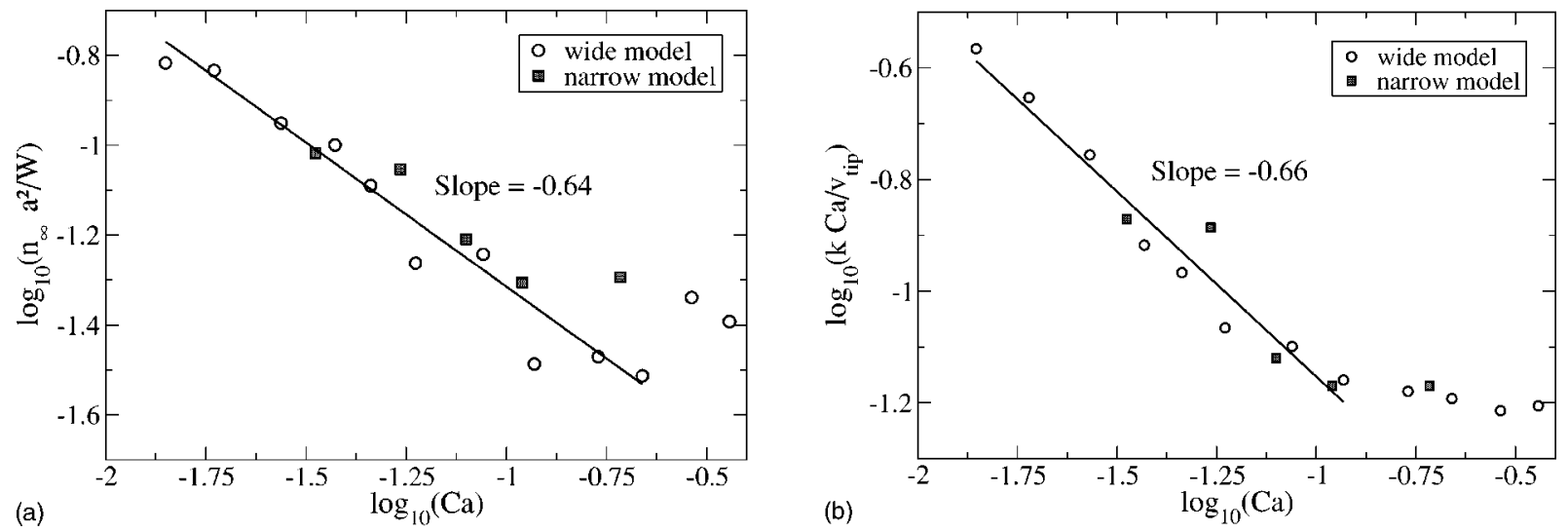

FIG. 9. Double logarithmic plots of (a) the saturated mass density $n_{\infty}$ and (b) of the speed of the most advanced finger, $v_{\text {tip }}$, as a function of the capillary number, for the two sets of experiments. Both plots are consistent with a scaling in the form $n_{\infty} \propto \mathrm{Ca} / v_{\text {tip }} \propto \mathrm{Ca}^{-\alpha}$, with $\alpha$ $\approx 0.65$.

creases. In the following we first present results relative to the "mass density" in the stagnant zone, $n_{\infty}$, and to the velocity of the most advanced finger tip, $v_{\text {tip. }}$ In the end we discuss the results relative to measurements of the characteristic width of the fingerlike structures, the definition of which is not as straightforward and clear as those of $n_{\infty}$ and $v_{\text {tip. }}$.

The evolution of the average mass density in the stagnant zone $n_{\infty}$ as a function of the capillary number is presented in Fig. 9(a) on a double-logarithmic scale. The data are consistent with a scaling law in the form $n_{\infty} \propto \mathrm{Ca}^{-\alpha}$, with a scaling exponent $\alpha \approx 0.65$ for both the wide and narrow models. Here $n_{\infty}$ has been measured by fitting the function $n_{\infty}[1$ $-\exp (-z / \lambda)]$ with both parameters free to our measured $n(z)$ data. As a result of the dependence of the mass of the invasion cluster on the capillary number [Fig. 9(a)], the speed of the most advanced finger tip, $v_{\text {tip }}$, is expected to depend on the filtration speed or capillary number in a nonlinear way. The saturated mass density and the speed of the most advanced finger are related to each other through Eq. (7), according to

$$
v_{\text {tip }} \propto \frac{\mathrm{Ca}}{n_{\infty}} .
$$

Based on that argument, $\mathrm{Ca} / v_{\text {tip }}$ should therefore scale in the same way as $n_{\infty}$ with respect to the capillary number. In Fig. 9 (b), the quantity $k \cdot \mathrm{Ca} / v_{\text {tip }}$ is plotted as a function of $\mathrm{Ca}$ on a double-logarithmic scale, where $k=1 \mathrm{~m} / \mathrm{s}$. The plot is consistent with the expected scaling (23) and the result for the mass density presented above.

The study of the dependence of the finger width on the capillary number is somewhat less straightforward, because our invading clusters structures exhibit extensive branching and display "fingers" both at small scales as "capillary fingers" and at large scales as "viscous fingers." Thus, a precise definition of a finger, and furthermore a finger width, is not an easy task for those structures. A possible method to determine the viscous finger width would consist in finding the characteristic crossover length between geometric features characteristic of viscous fingering and those characteristic of capillary fingering from the density-density correlation func- tion of the structures. However, as a result of the small difference in fractal dimension between the two regimes, $1.83 \pm 0.01[6,30]$ for capillary fingering and $1.62 \pm 0.04$ $[31,38]$ for viscous fingering, larger systems would be necessary for this method to be accurate enough. An experimental determination of the characteristic width $w_{f}$ for viscous fingers was previously obtained for imbibition experiments [32], for which the characteristic finger width can be defined and found in a more straightforward manner. The obtained scaling was $w_{f} \propto \mathrm{Ca}^{-0.5}$. In those imbibition experiments, the finger width $w_{f}$ was measured as the average length of cut segments perpendicular to the flow direction. This method can also be applied in our experiments, but due to the small scale fractal nature of the invasion front, trapping of wetting fluid inside the fingers and capillary fingering on small length scales, it is not obvious which length scales are being probed with this method. The results that we obtain are plotted as a function of the capillary number in Fig. 10. Clusters of wetting liquid trapped behind the displacement front have been removed from the picture prior to analysis. We then define the front width $w_{f}$ as the average over $z$ and time of

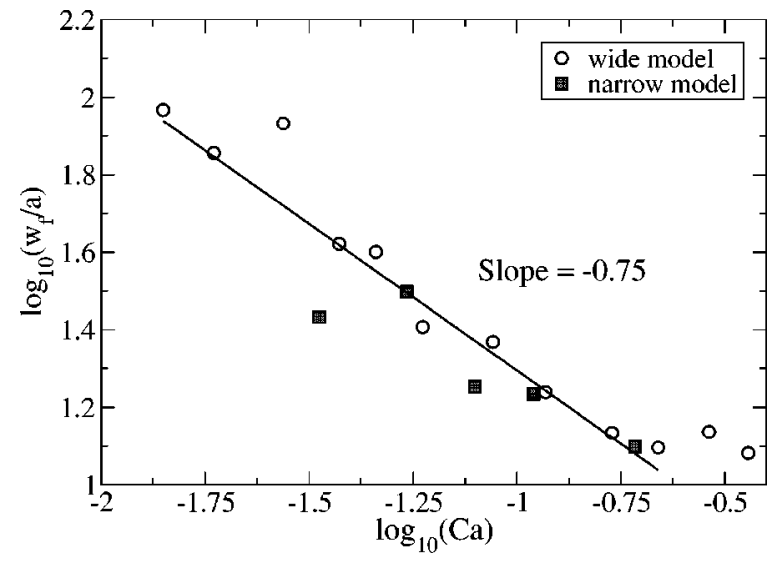

FIG. 10. Double-logarithmic plot of the measured characteristic width of the fingerlike structures as a function of the capillary number, for both the wide and narrow models. The data are consistent with a scaling of the finger width in the form $w_{f} \propto \mathrm{Ca}^{-\beta}$, with $\beta$ $\approx 0.75$. 
the length of the intersects between the invasion cluster emptied from these trapped regions and cuts perpendicular to the flow direction.

The measurements are consistent with a scaling law in the form $w_{f} \propto \mathrm{Ca}^{-\beta}$, with $\beta \approx 0.75$. This is significantly different from what was measured for imbibition. It also differs significantly from the scaling law expected from theoretical arguments for percolation in a destabilizing gradient $[39,40]$ for two-dimensional systems: $w_{f} \propto \mathrm{Ca}^{-\beta}$ with $\beta=0.57$. In our experiments, the destabilizing field (pressure) is highly inhomogeneous, which may explain why the behavior expected from the percolation in a gradient theory is not really observed.

From Figs. 9 and 10, the observed scalings appear to be valid for a limited range of capillary numbers. For high capillary numbers the observed scaling breaks down for $\mathrm{Ca}$ $\approx 0.2$, which corresponds to situations where the characteristic finger width has reached the one pore limit. At the other limit, for small capillary numbers it is not clear if we reach the lower limit in capillary number. But we expect that the observed scaling breaks down for capillary numbers smaller than the criteria given in Eq. (10), $\mathrm{Ca} \sim 10^{-3}$. As a result of this, it should be noted that the measured exponents $\alpha$ and $\beta$ only are meaningful for intermediate capillary numbers between the crossovers to the high- and low-capillary-number regimes and that the length scale for the low-capillarynumber crossover is system size dependent. Our measured values should therefore be considered as lower bounds for the exponents rather than correct values since inclusion of data points in the crossover regimes will lead to underestimated values for the exponents. For more precise measurements of these exponents experiments on larger systems are needed.

\section{DISCUSSION AND PROSPECTS}

Following a procedure similar to that used by Arneodo et al. [19] to study the statistical average properties of offlattice DLA simulations in a linear channel, we introduce an average occupancy map $\pi(x, z)$. Let $g(x, z)$ be the invader occupation function equal to 1 when the local pore is air filled or 0 when it is liquid filled. For any position $(x, z)$ (where $z$ is relative to the tip position), $\pi(x, z)$ corresponds to the average over all times, of the invader occupation function. Points closer than $W$ from the inlet are excluded from this average, since the geometry of the invading structure is expected to be strongly influenced by the central point injection technique in that zone. Similarly to the average DLA situation [19], this average occupancy map saturates to a maximum value $\pi_{\max }$ around the central line $x=W / 2$, at distances $z$ larger than $W$ behind the tip position. For $z>W$, $\pi(x, z)$ fluctuates around an average saturated occupancy $\pi(x)$, which is independent of $z$. This average saturated occupancy is approximated for each experiment by averaging the invader occupation function over all times and all $z>W$. The quantity $\pi(x)$ depends on the capillary number, as expected since by definition $\int_{0}^{W} \pi(x) d x \simeq a^{2} n_{\mathrm{Ca}}$.

However, the shape of this function, apart from a different $\pi_{\max }$, seems reasonably independent of the capillary number

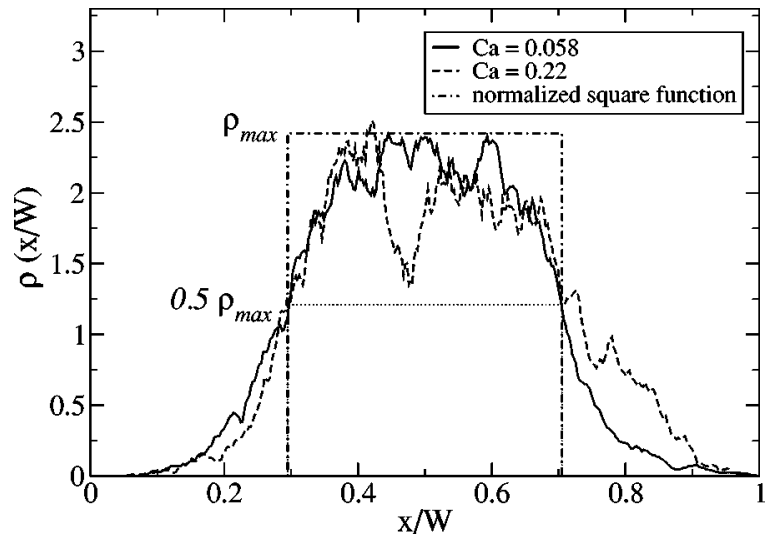

FIG. 11. Normalized average saturated occupation density for five experiments in the thin model at two different capillary numbers. Both functions present an effective width around $0.4 \mathrm{~W}$.

when $\mathrm{Ca}>0.03$. This is demonstrated in Fig. 11, where the normalized saturated occupancy function for the thin model, $\rho(x / W)=W \pi(x) / \int_{0}^{W} \pi(x) d x$, is displayed for five experiments at $\mathrm{Ca}=0.058$ and five experiments at $\mathrm{Ca}=0.22$. The characteristic width of this function is obtained by using two definitions introduced by Arneodo et al. [19], $\int_{0}^{W} \pi(x) d x / \pi_{\max }$ or $\left(x^{+}-x^{-}\right)$where $\pi\left(x^{+}\right)=\pi\left(x^{-}\right)=0.5 \pi_{\max }$. Both definitions for both capillary numbers lead to a characteristic width around $0.4 \mathrm{~W}$ (Fig. 11). The mean occupancy profile or average envelope, defined as the contour level $\pi(x, z)=0.5 \pi_{\max }$, presents some similarities to the shape of a Saffman-Taylor finger corresponding to the same saturation width [7]. Similar contour levels observed in off-lattice DLA simulations correspond to a characteristic selected width $0.62 W[19,20]$ and present some similarities with the corresponding Saffman-Taylor finger, although the detailed shape differs [20]. The pressure field recorded at some distance exceeding $0.2 \mathrm{~W}$ of the invader structure [41] is also consistently comparable to the pressure field around such a Saffman-Taylor finger, which arises from the fact that the Laplace equation controlling the pressure field is sensitive to the coarse external shape of the invader's boundary, rather than to the details of the branched structure inside this average envelope.

Inside the average envelopes, at intermediate scales the structures correspond to "DLA-like" viscous fingers. On these length scales the viscous pressure differences in the fluid are significant compared to the width of the capillary pressure thresholds distribution $W_{c}$. The structure is fractal [41], with a fractal dimension identical to that previously measured in viscous fingering experiments in random porous media similar to ours $1.62 \pm 0.04$ [31]. On length scales smaller than characteristic finger width $w_{f}$, the viscous pressure drops are small compared to $W_{c}$. Inside the fingers the structures correspond to capillary fingering with a fractal dimension $1.83 \pm 0.01$ [30]. The cutoff length $w_{f}$ between these two regimes is about the same size as the trapped wetting clusters. The saturation level of the envelope width seems insensitive to $\mathrm{Ca}$ when $\mathrm{Ca}>0.03$ [41]. This implies that the saturated mass dependence $\mathrm{Ca}$ displayed in Fig. 9 results from the decrease of the characteristic finger width $w_{f}$ with 
the capillary number (Fig. 10). The structures therefore look more branched and thin at higher capillary numbers (Fig. 2), even if they still occupy the same characteristic zone in the linear cells.

The similarity between viscous fingering and DLA [8] was first proposed by Paterson [15] and is based on the equivalence between the probability field for DLA and the pressure field in the viscous fingering case. They both seem to obey Laplace's equation with similar boundary condition, Eqs. (1) and (2). However, the characteristic of the intermediate scales in our viscous fingering experiments displays fundamental differences from DLA growth models: the obtained saturation width $0.4 \mathrm{~W}$ is significantly below the result $0.60 \mathrm{~W}$ obtained from off-lattice DLA simulations $[19,20]$ and also lower than the width $0.5 \mathrm{~W}$ obtained for on-lattice DLA simulations [19] or for a Saffman-Taylor finger in a empty Hele-Shaw cell at high capillary numbers [7]. Average widths higher than $0.5 \mathrm{~W}$ are reported in empty thin cells [18], depending on the capillary number and on the ratio of the cell thickness over width, which affects the role of a wetting oil film left behind the invasion front. The displacement regimes that we have studied correspond to what the authors of Ref. [18] would refer to with their notations as $1 / B \sim 1000-10000$ and $w / b=200$, for which their experiments would display selected widths larger than $0.5 \mathrm{~W}$ according to Fig. 3in their article [18]. The observed fractal dimension of the viscous fingering structures in a random porous medium similar to ours, $1.62 \pm 0.04$ [31], is also somewhat smaller than 1.7, the fractal dimension in radial viscous fingering in empty Hele-Shaw cells [17], in radial Laplacian growth [27], or in DLA [20,29]. This shows experimentally that there are some fundamental differences between these processes and viscous fingering in random porous media. Many works have recently focused on generalizations of Laplacian growth processes and DLA and put evidence on the fact that the fractal dimension or even the fractality of the grown structure depends on the precise boundary condition, growth rate, fraction of the perimeter growing simultaneously, and shot noise at the interface boundary [22-28]. The difference between these models shows the importance of the precise growth conditions along the interface. In the present experiments, the disordered porous material creates a nontrivial growth condition along the interface, dependent on the local pressure level, pressure gradient, and of a quenched disorder in the capillary pressure thresholds.

The fact that in these experiments the width of the mean occupancy profile and fractal dimension are below the DLA case make it more similar to another type of generalized DLA models-namely, dielectric breakdown models (DBM's). In the DBM the growth probability of the structures is proportional to a power higher than unity of the pressure gradient $[20,42]$. To better support the comparison to these various models, the precise determination of the average occupancy maps and fractal dimension(s) of the structure, as a function of capillary number and system size, is the subject of ongoing work [41].

\section{CONCLUSION}

We have studied the dynamics of the invasion process observed during drainage in a two-dimensional porous medium, for extraction speeds that result in an unstable fingering of the displacing nonwetting fluid into the displaced wetting fluid.

Our main finding is that for a given porous medium, the displacement is controlled by an invasion probability density that only depends on the distance of the point where it is measured to the tip of the most advanced finger tip and is independent of the capillary number. The decay of this invasion probability density, $\phi(z)$, defines an active zone for the invasion process, outside of which the viscous pressure field can be considered to be screened by the invasion structure. In particular, parts of the invasion structure lying outside this active zone are frozen and do not evolve in time any more. The size of the active zone, of characteristic screening length, $\lambda$, was found to be independent of the capillary number for a wide range of injection rates. In addition, experiments carried out on models with two different widths suggested that the invasion probability density appears to be capillary number independent, its actual shape being possibly fixed by the system size. While the invasion process is described by an invasion probability density that is independent of the capillary number, the invasion speed and displaced volume in the stagnant zone were found to scale on the capillary according to power laws, $n_{\infty} \propto \mathrm{Ca} / v_{\text {tip }} \propto \mathrm{Ca}^{-0.65}$.

Current work [41] was also reported on the mean occupancy density behind the most advanced tip, which also seems to be a function of shape independent of the capillary number as soon as $\mathrm{Ca}>0.03$, although its average value is capillary number dependent. The ratio of characteristic width of such density map over the system width was found around 0.4 , which is significantly below the result corresponding to DLA simulations (0.6).

The link between the growth probability and the pressure field has been studied. An expression for the pressure boundary condition relating the pressure on the interface of the invader to the growth probability density function on the cluster, $\phi(z)$, has been calculated. The measured pressure has been compared to the corresponding simulated pressure by solving the Laplace equation for the pressure field using this expression for the boundary condition on the cluster. Good agreement is found between the simulations and the experiments.

System size dependences should be subject to further investigations, both experimentally and by means of computer simulations.

\section{ACKNOWLEDGMENTS}

This work was supported by NFR, the Norwegian Research Council, VISTA, the Norwegian academy of science and Letters' research program with Statoil, and the French/ Norwegian collaboration PICS. 
[1] J. Bear, Dynamics of Fluids in Porous Media (American Elsevier, New York, 1972).

[2] F. A. L. Dullien, Porous Media Fluid Transport and Pore Structure, 2nd ed. (Academic, San Diego, 1992).

[3] M. Sahimi, Rev. Mod. Phys. 65, 1393 (1993).

[4] M. Sahimi, Flow and Transport in Porous Media and Fractured Rock (VCH Verlagsgesellschaft GmbH, Weinheim, Germany, 1995).

[5] R. Lenormand, E. Touboul, and C. Zarcone, J. Fluid Mech. 189, 165 (1988).

[6] R. Lenormand and C. Zarcone, Transp. Porous Media 4, 599 (1989).

[7] P. G. Saffman and G. Taylor, Proc. R. Soc. London, Ser. A 245, 312 (1958).

[8] T. A. Witten and L. M. Sander, Phys. Rev. Lett. 47, 1400 (1981).

[9] M. Plischke and Z. Racz, Phys. Rev. Lett. 53, 415 (1984).

[10] M. Plischke and Z. Racz, Phys. Rev. Lett. 54, 2054 (1985).

[11] P. Meakin, A. Coniglio, H. E. Stanley, and T. A. Witten, Phys. Rev. A 34, 3325 (1986).

[12] T. C. Halsey, P. Meakin, and I. Procaccia, Phys. Rev. Lett. 56, 854 (1986).

[13] C. Amitrano, A. Coniglio, and F. di Liberto, Phys. Rev. Lett. 57, 1016 (1986)

[14] B. B. Mandelbrot, J. Fluid Mech. 62, 331 (1974).

[15] L. Paterson, Phys. Rev. Lett. 52, 1621 (1984).

[16] B. Shraiman and D. Bensimon, Phys. Rev. A 30, 2840 (1984).

[17] E. Sharon, M. G. Moore, W. D. McCormick, and H. L. Swinney, Phys. Rev. Lett. 91, 205504 (2003).

[18] M. G. Moore, A. Juel, J. M. Burgess, W. D. McCormick, and H. L. Swinney, Phys. Rev. E 65, 030601 (2002).

[19] A. Arneodo, J. Elezgaray, M. Tabard, and F. Tallet, Phys. Rev. E 53, 6200 (1996).

[20] E. Somfai, R. C. Ball, J. P. DeVita, and L. M. Sander, Phys. Rev. E 68, 020401 (2003).

[21] L. M. Sander, P. Ramanlal, and E. Ben-Jacob, Phys. Rev. A 32, 3160 (1985).

[22] M. B. Hastings and L. S. Levitov, Physica D 116, 244 (1998).
[23] B. Davidovitch, H. G. E. Hentschel, Z. Olami, I. Procaccia, L. M. Sander, and E. Somfai, Phys. Rev. E 59, 1368 (1999).

[24] F. Barra, B. Davidovitch, A. Levermann, and I. Procaccia, Phys. Rev. Lett. 87, 134501 (2001).

[25] F. Barra, B. Davidovitch, and I. Procaccia, Phys. Rev. Lett. 65, 046144 (2002).

[26] H. G. E. Hentschel, A. Levermann, and I. Procaccia, Phys. Rev. E 66, 016308 (2002).

[27] A. Levermann and I. Procaccia, Phys. Rev. E 69, 031401 (2004).

[28] M. G. Stepanov and L. S. Levitov, Phys. Rev. E 63, 061102 (2001).

[29] B. Davidovitch, A. Levermann, and I. Procaccia, Phys. Rev. E 62, R5919 (2000).

[30] R. Lenormand and C. Zarcone, Phys. Rev. Lett. 54, 2226 (1985).

[31] K. J. Måløy, J. Feder, and T. Jøssang, Phys. Rev. Lett. 55, 2688 (1985).

[32] J. P. Stokes, D. A. Weitz, J. P. Gollub, A. Dougherty, M. O. Robbins, P. M. Chaikin, and H. M. Lindsay, Phys. Rev. Lett. 57, 1718 (1986).

[33] D. A. Weitz, J. P. Stokes, R. C. Ball, and A. P. Kushnick, Phys. Rev. Lett. 59, 2967 (1987).

[34] R. Lenormand, C. Zarcone, and A. Sarr, J. Fluid Mech. 135, 337 (1983).

[35] R. Lenormand, J. Phys.: Condens. Matter 2, SA79 (1990).

[36] Y. Méheust, G. Løvoll, K. J. Måløy, and J. Schmittbuhl, Phys. Rev. E 66, 051603 (2002).

[37] G. G. Batrouni and A. Hansen, J. Stat. Phys. 52, 747 (1988).

[38] J.-D. Chen and D. Wilkinson, Phys. Rev. Lett. 55, 1892 (1985).

[39] R. Lenormand, Proc. R. Soc. London, Ser. A 423, 159 (1989).

[40] Y. C. Yortsos, B. Xu, and D. Salin, Comput. Geosci. 5, 257 (2001).

[41] R. Toussaint, G. Løvoll, Y. Méheust, K. J. Måløy, and J. Schmittbuhl (unpublished).

[42] J. Mathiesen and M. H. Jensen, Phys. Rev. Lett. 88, 235505 (2002). 